\title{
Molecular basis for the expression of major vault protein induced by hyperosmotic stress in SW620 human colon cancer cells
}

\author{
YUSUKE TAJITSU $^{1}$, RYUJI IKEDA ${ }^{1}$, YUKIHIKO NISHIZAWA ${ }^{1}$, HIROFUMI MATAKI $^{1}$, XIAO-FANG CHE $^{2}$, \\ TOMOYUKI SUMIZAWA ${ }^{3}$, MINA NITTA ${ }^{1}$, TATSUYA YAMAGUCHI ${ }^{1}$, MASATATSU YAMAMOTO $^{2}$, SHO TABATA $^{4}$, \\ SHIN-ICHI AKIYAMA ${ }^{4}$, KATSUSHI YAMADA ${ }^{5}$, TATSUHIKO FURUKAWA ${ }^{2}$ and YASUO TAKEDA ${ }^{1}$ \\ Departments of ${ }^{1}$ Clinical Pharmacy and Pharmacology, and ${ }^{2}$ Molecular Oncology, Graduate School of Medical \\ and Dental Sciences, Kagoshima University, Kagoshima 890-8520; ${ }^{3}$ Department of Domestic Science, \\ Kagoshima Women's College, Kagoshima 890-8565; ${ }^{4}$ Department of Respiratory Medicine and Rheumatology, \\ Institute of Health Biosciences, The University of Tokushima Graduate School, Tokushima 770-8503; \\ ${ }^{5}$ Department of Clinical Pharmacology, Faculty of Pharmaceutical Sciences, \\ Nagasaki International University, Nagasaki 859-3298, Japan
}

Received November 5, 2012; Accepted January 9, 2013

DOI: $10.3892 /$ ijmm.2013.1428

\begin{abstract}
Major vault protein (MVP) is identical to lung resistance-related protein (LRP), which is the major component of vaults. Vaults are considered to play a protective role against xenobiotics and other types of stress. In a previous study, we reported that the expression levels of MVP in SW620 human colon cancer cells were increased in hypertonic culture medium with sucrose. However, the molecular mechanism behind the induction of MVP expression by osmotic stress has not yet been elucidated. Therefore, in the present study, we investigated the mechanism behind the induction of MVP expression by osmotic stress. Under hyperosmotic stress conditions, the ubiquitination of specificity protein 1 (Sp1) decreased, Spl protein levels increased, its binding to the MVP promoter was enhanced, and small interfering RNA (siRNA) for Sp1 suppressed the induction of MVP expression. The inhibition of c-jun N-terminal kinase (JNK) by SP600125, a specific JNK inhibitor, decreased the expression of MVP and $\mathrm{Sp} 1 \mathrm{under}$ hyperosmotic conditions. Our data indicate that the stabilization and upregulation of $\mathrm{Sp} 1$ protein expression by JNK participate in the inhibition of the ubiquitination and degradation of Sp1, and thus in the induction of MVP expression under hyperosmotic conditions.
\end{abstract}

Correspondence to: Professor Yasuo Takeda, Department of Clinical Pharmacy and Pharmacology, Graduate School of Medical and Dental Sciences, Kagoshima University, 8-35-1 Sakuragaoka, Kagoshima 890-8520, Japan

E-mail:takeda@m.kufm.kagoshima-u.ac.jp

Key words: hyperosmotic stress, major vault protein, specificity protein 1 , c-jun $\mathrm{N}$-terminal kinase, ubiquitination

\section{Introduction}

Vaults are predominantly cytoplasmic ribonucleoprotein particles identified in preparations of coated vesicles (1). The vault complex has a barrel-like structure with an invaginated waist and two protruding caps. Mammalian vaults are composed of major vault protein (MVP) and two minor vault proteins [vault poly(ADP-ribose)polymerase (VPARP) and telomerase-associated protein 1 (TEP1)] as well as small untranslated RNAs (2). Vault particles have been highly conserved throughout evolution and are found in numerous eukaryotic species (3). These data suggest that vaults have an important cellular function. However, the precise function of vault particles has not yet been completely elucidated.

MVP is the main component of vault particles and covers over $70 \%$ of the particle mass. Since MVP is identical to lung resistance-related protein (LRP), which was first identified in a non-P-gp multidrug-resistant cancer cell line and has been shown to be overexpressed in several multidrug resistant cell lines $(4,5)$, it has been suggested that MVP is involved in multidrug resistance.

On the other hand, a number of previous studies have reported that MVP expression is induced by a variety of cellular stresses such as DNA damaging agents, UV irradiation, hypoxia and hyperthermia (6-8). Kowalski et al (9) reported that MVP is involved in host resistance to infection with Pseudomonas aeruginosa. Ryu et al (10) demonstrated that MVP enhanced the resistance of cells to apoptosis induced by $\mathrm{H}_{2} \mathrm{O}_{2}$ in senescent human diploid fibroblasts (HDFs). These findings suggest that MVP plays an important role in cellular responses to stress.

It has previously been reported that the human MVP promoter lacks a TATA-box, as well as other core promoter elements, but harbors putative transcriptional factor binding sites for p53, STAT1, MyoD, specificity protein 1 (Sp1), GATA and YB-1 (11). Transcription factors are involved in the regulation of MVP in different cell types $(6-9,12,13)$. We previously 
reported that hyperosmotic stress upregulated the expression of MVP (14). However, it is unclear which transcription factor affects MVP expression under hyperosmotic stress conditions and the underlying mechanism has not yet been elucidated. Therefore, the aim of this study was to investigate the mechanism behind the induction of MVP expression under hyperosmotic stress conditions.

\section{Materials and methods}

Cell line, antibodies and chemicals. The SW620 human colon carcinoma cell line was provided by Dr A.T. Fojo (National Cancer Institute, Bethesda, MD, USA). HA-tagged ubiquitin for constructing the plasmid was provided by Dr Dirk Bohmann (European Molecular Biology Laboratory, Heidelberg, Germany). RPMI-1640 medium was purchased from Nissui Seiyaku Co. (Tokyo, Japan). Fetal calf serum (FCS) was obtained from JRH Biosciences (Lenexa, KS, USA). Sucrose was obtained from Wako Pure Chemical Industries, Ltd. (Osaka, Japan). A rabbit anti-ERK polyclonal antibody (C-16), mouse anti-Sp1 monoclonal antibody (E-3), rabbit anti-Sp1 polyclonal antibody (PEP2), rabbit anti-HA polyclonal antibody (Y-11) and mouse phosphothreonin monoclonal antibody (H-2) were obtained from Santa Cruz Biotechnology, Inc. A mouse anti-STAT1 monoclonal antibody (610185) was obtained from BD Biosciences. A rabbit anti-MVP polyclonal antibody was prepared using a glutathione S-transferase (GST)-MVP (aa 694-794) fusion protein as an antigen (5).

Immunoblot analysis. Immunoblot analysis was carried out as described previously $(5,17)$.

Reverse transcription-polymerase chain reaction (RT-PCR). Total RNA from cultured cells was isolated using TRIzol reagent (Invitrogen). RT-PCR was performed with the SuperScript One-Step RT-PCR system and gene-specific primers according to the manufacturer's instructions (Invitrogen). Reaction mixtures contained total RNA (500 ng of each), $0.2 \mathrm{mM}$ dNTPs, $0.2 \mu \mathrm{M}$ of $\mathrm{Spl}$ primer (sense, 5'-TGGAAGCAGCTGAGGCAATGG-3' and antisense, 5'-ATCCAGCCTCAGCTAGTTCAA-3') and GAPDH primer (sense, 5'-AGAACATCATCCCTGCCTCTA CTGG-3' and antisense, 5'-AAAGGTGGAGGAGTGGGTGT CGCTG-3'), enzyme mixture containing SuperScript II RT, Platinum TaqDNA polymerase, and $1 \mathrm{X}$ buffer with $1.2 \mathrm{mM}$ $\mathrm{MgSO}_{4}$. The reaction was performed at $50^{\circ} \mathrm{C}$ for $20 \mathrm{~min}, 94^{\circ} \mathrm{C}$ for $2 \mathrm{~min}$, followed by 28 cycles of $94^{\circ} \mathrm{C}$ for $15 \mathrm{sec}, 55^{\circ} \mathrm{C}$ for $30 \mathrm{sec}$ and $70^{\circ} \mathrm{C}$ for $30 \mathrm{sec}$.

Dual-luciferase reporter assay. The generation of the MVP promoter-luciferase construct (pMVP78) has been described in a previous study of ours (6). The luciferase assay was performed using the Dual-Luciferase Reporter Assay System according to the manufacturer's instructions (Promega). In order to perform the luciferase assay, transfected SW620 cells were cultured in the presence of sucrose. Cells were washed with PBS and lysed using $100 \mu \mathrm{l}$ of passive lysis buffer per well. Luminescence assays were performed using a luminometer (TD-20/20 Luminometer; Turner Designs, Sunnyvale, CA, USA). All experiments were performed in triplicate and the results were normalized to pRL-TK activity.
RNA interference. STAT1 siRNA was obtained from Cell Signaling Technology (no. 6544). siRNA duplexes were synthesized using the Silencer ${ }^{\mathrm{TM}}$ siRNA construction kit (Ambion Inc., Austin, TX, USA). The siRNA used in this study consisted of a 21-nucleotide sense strand and a 21-nucleotide antisense strand with a two-nucleotide overhang at the 3'-end. Sequences were as follows: $G F P$-siRNA target sequence, 5'-AAGCGTTCAACTA GCAGACCA-3'; Spl-siRNA target sequence, 5'-AAGGAA CAGAGTGGCAGCAGT-3'. siRNA (100 nM) was introduced into the SW620 cells using Lipofectamine ${ }^{\mathrm{TM}} 2000$ according to the manufacturer's instructions (Invitrogen).

In vitro ubiquitination assays. COS-7 cells were transfected with expression vectors encoding HA-tagged ubiquitin for $24 \mathrm{~h}$ after transfection, and were exposed for $12 \mathrm{~h}$ to normal osmotic or hyperosmotic conditions in the presence of the proteasome inhibitor, MG132 $(5 \mu \mathrm{M})$. The cells were then lysed in RIPA buffer [50 mM Tris- $\mathrm{HCl}$ (pH 8.0), $150 \mathrm{mM} \mathrm{NaCl}, 1 \%$ Nonidet P-40 (NP-40), 0.1\% SDS, $0.5 \%$ sodium deoxycholate, $1 \mathrm{mM}$ p-amidinophenyl methanesulfonyl fluoride hydrochloride (APMSF) and $1 \mathrm{mg} / \mathrm{ml}$ aprotinin] and lysates (500 $\mu \mathrm{g}$ protein) were immunoprecipitated with anti-Sp1 antibody followed by immunoblotting with anti-HA antibody.

Immunoprecipitation. Cells were lysed with RIPA buffer and centrifuged at 7,500 rpm for $10 \mathrm{~min}$ at $4^{\circ} \mathrm{C}$. Subsequent to protein extraction, $500 \mu \mathrm{g}$ of total cell lysates were incubated with anti-Sp1 monoclonal antibody at $4^{\circ} \mathrm{C}$ for $1 \mathrm{~h}$. A total of $30 \mu \mathrm{l}$ of a $50 \%$ slurry of protein G-Sepharose 4B in RIPA buffer was then added to the reaction mixtures and incubated for $12 \mathrm{~h}$ at $4^{\circ} \mathrm{C}$ with rotation. Following rapid centrifugation, the resulting pellets were washed three times with RIPA buffer, and the immunoprecipitated proteins were analyzed by immunoblotting using anti-phosphothreonin antibody or anti-Sp1 polyclonal antibody.

Chromatin immunoprecipitation (ChIP) assay. Cells were fixed with $1 \%$ formaldehyde for $10 \mathrm{~min}$ at $37^{\circ} \mathrm{C}$ to cross-link protein to DNA. A chromatin immunoprecipitation (ChIP) assay was carried out using a ChIP assay kit (Upstate Biotechnology), according to the manufacturer's instructions. The soluble DNA fraction was mixed with an anti-Sp1 polyclonal antibody or non-immunized rabbit IgG (Santa Cruz Biotechnology), and the precipitated DNA was amplified with primers for the MVP promoter [5'-GCCAGCTGGCTCCAAGGTAG-3' (sense) and 5'-ATCACTTCCCGGCAGGGCAA-3' (antisense)].

\section{Results}

Spl directly regulates the transcription of the MVP gene under hyperosmotic stress conditions. We previously reported that hyperosmotic stress upregulates $M V P$ promoter activity. To identify important MVP promoter elements that are involved in the induction of MVP expression by hyperosmotic stress, we generated luciferase reporter constructs containing various promoter elements. The pMVP78 construct contained three elements, Sp1, p53 and STAT, which were reported as transcription factors involved in $M V P$ promoter activity (Fig. 1A). Following the transfection of the plasmids into the SW620 cells, cells were subjected to hyperosmotic stress and 
A

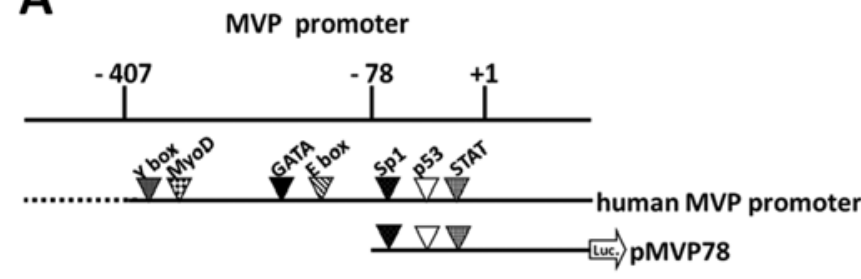

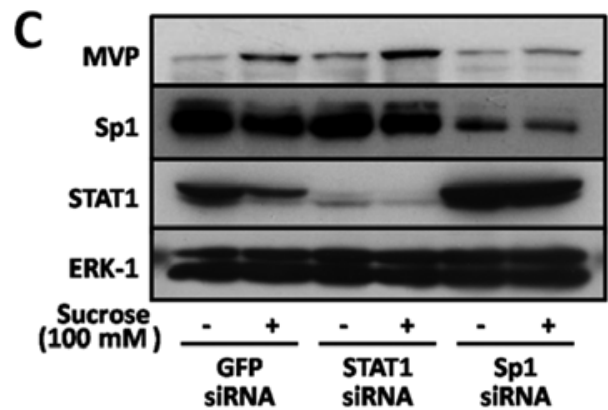

D

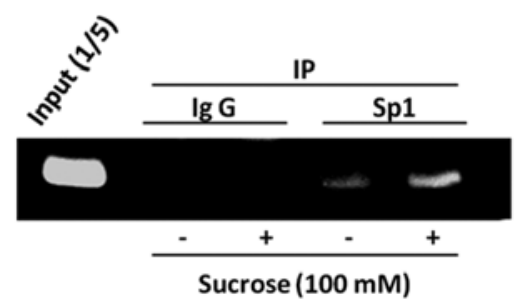

Figure 1. (A) Outline of the MVP-promoter constructs used. The top line indicates the promoter region (-407) upstream of the MVP transcription start site $(+1)$. The pMVP78 construct harbors the Sp1, p53 and STAT binding sites. This construct was linked to a luciferase reporter gene (luc). (B) SW620 cells were transiently transfected with pMVP78 (800 ng) and pRL-TK (200 ng) reporter gene constructs using Lipofectamine 2000 reagent. After transfection, the cells were incubated in the presence $(+)$ or absence $(-)$ of $100 \mathrm{mM}$ sucrose for $48 \mathrm{~h}$ and then assayed for luciferase activity. Experiments were performed in triplicate and the results were normalized to pRL-TK activity. Bars indicated the means \pm SD. ${ }^{*} \mathrm{P}<0.05$. (C) SW620 cells were transfected with siRNA targeting Sp1, STAT1, or GFP and cultured in $100 \mathrm{mM}$ sucrose for $48 \mathrm{~h}$, and cell lysates were tehn prepared from these cells. The expression of Sp1, STAT1, MVP and ERK-1 was detected by immunoblot analsyis using anti-Sp1, anti-STAT1, anti-MVP and anti-ERK-1 antibodies, respectively. (D) The SW620 cells were treated with $100 \mathrm{mM}$ sucrose for $72 \mathrm{~h}$. Cells were fixed with formaldehyde to form a DNA-protein complex and were subjected to ChIP assay, as described in Materials and methods. A PCR primer for the MVP promoter was used to detect the promoter fragments in the immunoprecipitates. The input lane represents $20 \%$ of the total chromatin used in the ChIP assay.

MVP promoter activity was monitored by luciferase assay. Compared to basal luciferase activity, luciferase activity was approximately 2-fold higher in the pMVP78-transfected cells treated with sucrose (Fig. 1B). These results suggest that Sp1, p53, or STAT contribute to the enhancement of $M V P$ promoter activity by hyperosmotic stress.

SW620 cells have a mutation in the p53 gene (codon 273, Arg $\rightarrow$ His). This suggests that Sp1 or STAT1 is involved in the induction of MVP expression by hyperosmotic stress. To determine whether Sp1 or STAT1 are involved in the induction of MVP by hyperosmotic stress, SW620 cells were transfected with siRNA targeting Sp1 or STAT1.Sp1 knockdown inhibited the induction of MVP expression by hyperosmotic stress in the SW620 cells (Fig. 1C). However, STAT1 knockdown did not affect the induction of MVP expression. These results suggest that Sp1 is involved in the induction of MVP expression by hyperosmotic stress in SW620 cells.

To determine whether Sp1 can bind to the promoter region of the MVP gene in SW620 cells under hyperosmotic stress conditions, we performed a chromatin immunoprecipitation (ChIP) assay using anti-Sp1 antibody. The presence of the MVP promoter in chromatin immunoprecipitates was assessed by PCR using a specific pair of primers spanning the Spl-bind site in the $M V P$ gene promoter. Spl recruitment to the $M V P$ promoter was enhanced by hyperosmotic stress in SW620 cells (Fig. 1D). These results indicate that $\mathrm{Sp} 1$ directly regulates the transcription of the MVP gene under hyperosmotic stress conditions.
Hyperosmotic stress upregulates Spl protein levels. To determine whether hyperosmotic stress affects the expression of $\mathrm{Spl}$, we performed immunoblot analysis using anti-Spl antibody. Fig. 2A shows that the expression of Sp1 increased with increasing concentrations of sucrose from 50 to $200 \mathrm{mM}$. Sp1 protein levels increased in a time-dependent manner during treatment with sucrose at $100 \mathrm{mM}$, and achieved a peak at $12 \mathrm{~h}$ (Fig. 2B).

We then examined the effect of hyperosmotic stress on the expression levels of Spl mRNA in SW620 cells. Hyperosmotic stress did not affect Spl mRNA levels (Fig. 2C and D). These results suggest that hyperosmotic stress upregulates $\mathrm{Spl}$ protein levels by reducing the turnover rate.

Hyperosmotic stress suppresses proteasome-dependent Spl degradation by inhibiting ubiquitination. To examine the possibility that the accumulation of Sp1 protein reflects a reduction in its degradation rate, we investigated whether the induction of $\mathrm{Sp1}$ expression by hyperosmotic stress occurs in SW620 cells exposed to cycloheximide.

As shown in Fig. 3A, cycloheximide did not inhibit the induction of $\mathrm{Sp1}$ expression under hyperosmotic conditions. This result suggests that the degradation of Sp1 may be suppressed under hyperosmotic conditions. We therefore investigated the effect of MG132, a proteasome inhibitor, on Sp1 protein levels. Proteasome inhibition in the SW620 cells by MG132 increased Sp1 protein levels (Fig. 3B). To determine 
A

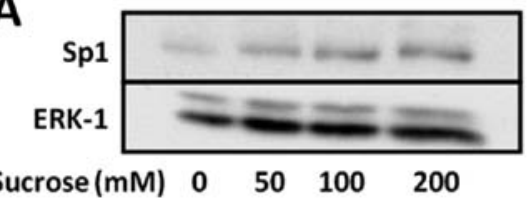

B

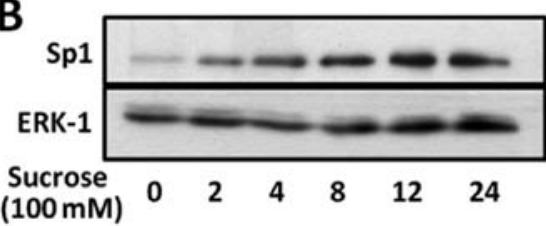

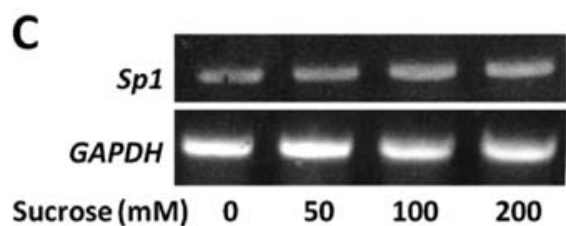

D

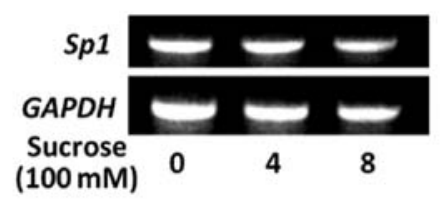

Figure 2. Effect of osmotic stress on Sp1 expression levels in SW620 cells. (A and B) SW620 cells were incubated in (A) 0-200 mM sucrose for 8 h, or (B) $100 \mathrm{mM}$ sucrose for $24 \mathrm{~h}$. After incubation, cell lysates were prepared and analyzed for Sp1 and ERK-1 protein expression by immunoblot analysis using anti-Sp1 or anti-ERK-1 antibodies. (C and D) SW620 cells were incubated in (C) 0-200 mM sucrose for $8 \mathrm{~h}$, or (D) $100 \mathrm{mM}$ sucrose for $8 \mathrm{~h}$. After incubation, cell lysates were prepared and analyzed for Spl mRNA by RT-PCR. The expression of GAPDH was used as the loading control.

A

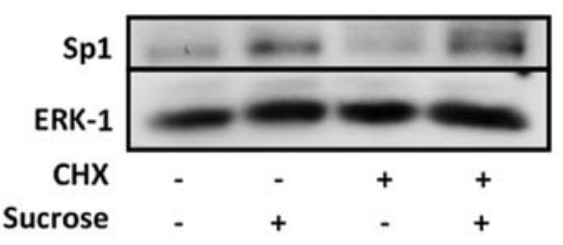

B

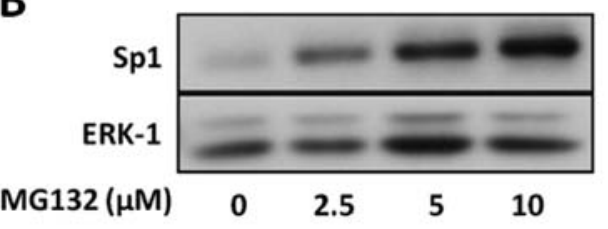

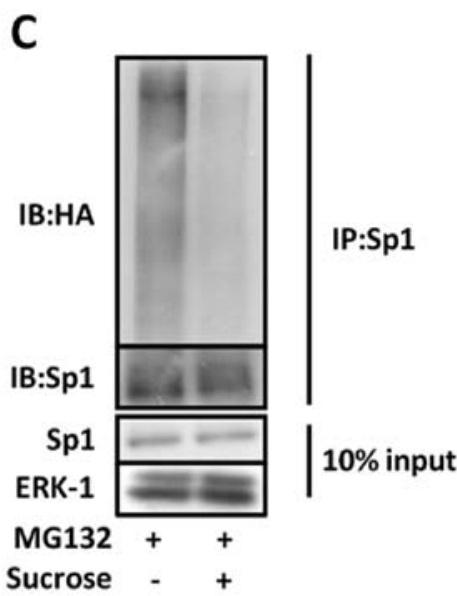

Figure 3. (A) SW620 cells were treated with sucrose in the presence or absence of cyclohexymide. Cell lysates were prepared from these cells. The expression of Sp1 and ERK-1 was detected by immunoblot analysis using antibodies against Sp1 or ERK-1. (B) Effect of the proteasome inhibitor, MG132, on the expression of Sp1 in SW620 cells. SW620 cells were incubated in 0-10 $\mu$ M MG132. Cell lysates were prepared from these cells. The expression of Sp1 and ERK-1 was detected by immunoblot analysis using antibodies against Sp1 or ERK-1. (C) COS-7 cells were transfected with a vector encoding HA-tagged ubiquitin. Transfected cells were treated with sucrose for $12 \mathrm{~h}$ in the presence or absence of MG132 (5 $\mu \mathrm{M})$. Ubiquitinated proteins were immunoprecipitated with antiSp1 antibody, and ubiquitinated Sp1 was detected by immunoblot analysis using anti-HA antibody.

whether hyperosmotic stress affects the ubiquitination of Sp1, COS-7 cells were transfected with HA-tagged ubiquitin. The transfected cells were then incubated for $12 \mathrm{~h}$ under hyperosmotic conditions in the presence or absence of MG132. Ubiquitinated Sp1 was detected by immunoblot analsyis using anti-HA antibody following immunoprecipitation with antiSp1 antibody. Furthermore, as shown in Fig. 3C, hyperosmotic stress suppressed the ubiquitination of $\mathrm{Sp} 1$. These results suggest that hyperosmotic stress induces the accumulation of Sp1 by suppressing its ubiquitination.

Induction of Spl is mediated by the activation of c-Jun $N$-terminal kinase (JNK) activation under hyperosmotic stress conditions. Hyperosmotic stress activates JNK, which plays an important role in cellular stress responses. A recent study showed that threonine phosphorylation by JNK-1 regulates the stability of Sp1 (15). In order to investigate whether JNK is activated in SW620 cells under hyperosmotic stress condi- tions, we analyzed the phosphorylation of JNK in SW620 cells under hyperosmotic stress conditions. The phosphorylation of JNK increased in a manner dependent on the sucrose concentration, which was increased from 50 to $200 \mathrm{mM}$ (Fig. 4A). These data suggest that hyperosmotic stress activates JNK in SW620 cells.

Therefore, we investigated the threonine phosphorylation of Sp1 under hyperosmotic stress conditions to confirm that hyperosmotic stress affects the threonine phosphorylation of Sp1 in SW620 cells. Hyperosmotic stress increased the threonine phosphorylation of Sp1 (Fig. 4B). Moreover, as shown in Fig. 4C, SP600125, a specific JNK inhibitor, inhibited the phosphorylation of Sp1 under hyperosmotic conditions.

To investigate whether JNK regulates the induction of Sp1 and MVP by hyperosmotic stress, SW620 cells were treated with SP600125 in the presence or absence of hyperosmotic stress. SP600125 inhibited the induction of Sp1 expression and consequently that of MVP under hyperosmotic stress condi- 
A

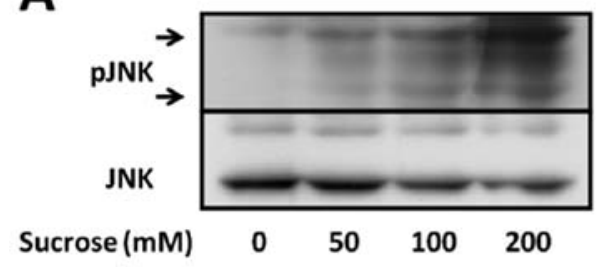

B

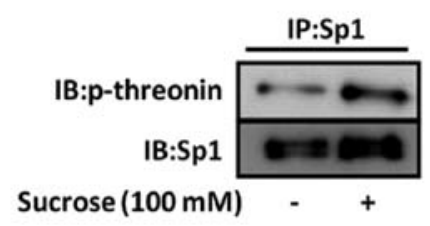

C

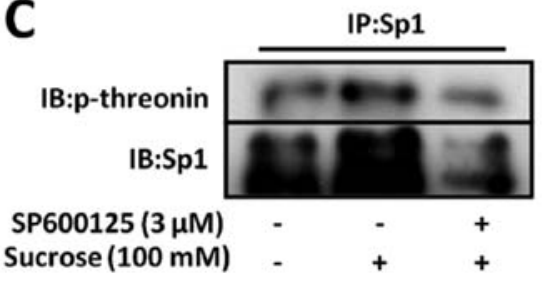

D

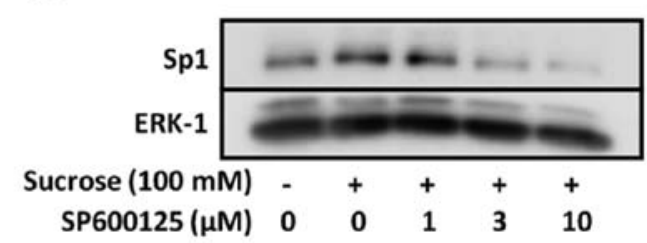

$\mathbf{E}$

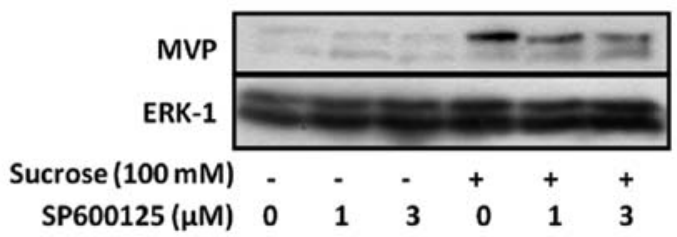

Figure 4. (A) SW620 cells were incubated in 0-200 mM sucrose for $1 \mathrm{~h}$, lysed, and analyzed for phosphorylated JNK and JNK by immunoblot analysis using anti-phosphorylated JNK or anti-JNK antibodies. (B) SW620 cells were incubated in $100 \mathrm{mM}$ sucrose for $12 \mathrm{~h}$. Phosphorylated proteins were immunoprecipitated with anti-Sp1 antibody, and phospho-threonine levels of Sp1 were detected by immunoblot analysis with anti-phospho-threonine antibody. (C) SW620 cells were treated with $100 \mathrm{mM}$ sucrose in the presence or absence of SP600125. After incubation, phosphorylated proteins were immunoprecipitated with anti-Sp1 antibody, and phospho-threonine levels of Sp1 were detected by immunoblot analysis with anti-phospho-threonine antibody. (D and E) SW620 cells were treated with $100 \mathrm{mM}$ sucrose in the presence or absence of SP600125. After incubation, cell lysates were prepared and analyzed for (D) Sp1 and (E) MVP by immunoblot analysis. Blotting of ERK-1 was used as the loading control.

tions (Fig. 4D and E). These data suggest that JNK upregulates MVP expression by inhibiting Sp1 degradation under hyperosmotic stress conditions. Therefore, the stability of Sp1 may be mediated by the enhancement of Sp1 phosphorylation by JNK under hyperosmotic stress conditions.

\section{Discussion}

One of the cellular stresses to which cells are exposed to in vivo is hyperosmotic stress. It has previously been reported that hyperosmotic stress induces apoptosis of cells (16). We previously reported that hyperosmotic stress modulates the PI3K/Akt pathway and induces MVP, which exerts a cytoprotective effect against apoptosis, thereby preventing cells from undergoing apoptosis by the activation of Akt in SW620 colon cancer cells (14). However, the molecular basis for the expression of MVP induced by hyperosmotic stress is unclear. Therefore, in the current study, we elucidated that $\mathrm{Sp} 1$ and JNK regulate the expression of MVP under hyperosmotic conditions.

Our results showed that hyperosmotic stress enhanced the binding of Spl to the MVP promoter and upregulated the transcription of the $M V P$ gene. Sp1 is ubiquitous in mammalian cells and is known as a housekeeping gene and a transcription factor with a $\mathrm{C} 2 \mathrm{H} 2$ zinc finger DNA binding domain, and also modulates transcription of numerous genes (17). It has been reported that $\mathrm{Sp} 1$ is activated by osmotic changes and regulates the transcription of a number of genes $(18,19)$.
Our data are consistent with these data from previous reports. Moreover, it has also been reported that the knockdown of Sp1 enhances the $\mathrm{H}_{2} \mathrm{O}_{2}$-induced apoptosis of U2OS osteosarcoma cells (20). We previously reported that MVP prevents cells from undergoing apoptosis (14). Our findings, as well as those from previous reports suggest that Sp1 augments the expression of MVP and consequently protects cells from apoptosis under hyperosmotic conditions.

JNK belongs to the family of mitogen-activated protein kinases (MAPKs) and is activated by several types of cellular stress, such as hypoxia and osmotic stress $(21,22)$. This study demonstrates that the inhibition of the ubiquitination and degradation of Sp1 by JNK causes the stabilization and upregulation of Sp1 protein, which participates in the induction of MVP expression under hyperosmotic stress conditions. Chuang et al (15) reported that the phosphorylation by JNK-1 regulates the stability of transcription factor $\mathrm{Spl}$ during mitosis. We previously reported that the expression levels of MVP in S1 small cell lung cancer cells increased in hypertonic culture medium with sucrose (14). As Spl protein levels also increased in $\mathrm{S} 1$ cells (data not shown), JNK may regulate the expression of MVP under hyperosmotic stress conditions in other cell lines. Recent studies have shown that JNK suppresses the interaction between Sp1 and ring finger protein 4 (RNF4), which is an E3 ubiquitin ligase of Sp1 $(15,23)$. These data suggest that JNK is activated by hyperosmotic stress and may suppress the interaction between Sp1 and RNF4 by phosphorylating the threonine residue of Sp1. Consistent with these data, the results from our 
study showed that hyperosmotic stress enhanced the threonine phosphorylation of Sp1 and decreased the ubiquitination of Sp1 (Figs. 3C and 4B).

We previously reported that p38 MAPK, which belongs to the family of MAPKs and is activated by cellular stress, is partly involved in the expression of MVP induced by hyperosmolytes. However, SB203580, a p38 MAPK-specific inhibitor, did not affect the induction of $\mathrm{Spl}$ by hyperosmotic stress (data not shown). These data suggest that p38 MAPK activates Sp1 without affecting the expression of Sp1 under hyperosmotic conditions. In fact, certain studies have shown that p38 MAPK activates $\mathrm{Sp1}(24,25)$. Further studies are required to define the role of p38 MAPK under hyperosmotic conditions.

In conclusion, the expression of MVP is upregulated by several types of cellular stress. The data from our study indicate that hyperosmotic stress upregulates $\mathrm{Sp1}$ expression levels by inhibiting ubiquitination through the activation of JNK, and the induction of Sp1 expression directly enhances MVP transcription.

\section{Acknowledgements}

We thank Dr Dirk Bohmann (European Molecular Biology Laboratory, Heidelberg, Germany) for providing the HA-tagged ubiquitin plasmid.

\section{References}

1. Kedersha NL and Rome LH: Isolation and characterization of a novel ribonucleoprotein particle: large structures contain a single species of small RNA. J Cell Biol 103: 699-709, 1986.

2. Kickhoefer VA, Vasu SK and Rome LH: Vaults are the answer, what is the question? Trends Cell Biol 6: 174-178, 1996.

3. Kedersha NL, Miquel MC, Bittner D and Rome LH: Vaults. II. Ribonucleoprotein structures are highly conserved among higher and lower eukaryotes. J Cell Biol 110: 895-901, 1990.

4. Scheffer GL, Wijngaard PL, Flens MJ, Izquierdo MA, Slovak ML, Pinedo HM, Meijer CJ, Clevers HC and Scheper RJ: The drug resistance-related protein LRP is the human major vault protein. Nat Med 1: 578-782, 1995.

5. Kitazono M, Sumizawa T, Takebayashi Y, Chen ZS, Furukawa T, Nagayama S, Tani A, Takao S, Aikou T and Akiyama S: Multidrug resistance and the lung resistance-related protein in human colon carcinoma SW-620 cells. J Natl Cancer Inst 91: $1647-1653,1999$.

6. Shimamoto Y, Sumizawa T, Haraguchi M, Gotanda T, Jueng HC, Furukawa T, Sakata R and Akiyama S: Direct activation of the human major vault protein gene by DNA-damaging agents. Oncol Rep 15: 645-652, 2006.

7. Stein U,Jürchott K, Schläfke M and Hohenberger P: Expression of multidrug resistance genes MVP, MDR1, and MRP1 determined sequentially before, during, and after hyperthermic isolated limb perfusion of soft tissue sarcoma and melanoma patients. J Clin Oncol 20: 3282-3292, 2002.

8. Iwashita K, Ikeda R, Takeda Y, Sumizawa T, Furukawa T, Yamaguchi T, Akiyama S and Yamada K: Major vault protein forms complexes with hypoxia-inducible factor (HIF)-1alpha and reduces HIF-1alpha level in ACHN human renal adenocarcinoma cells. Cancer Sci 101: 920-926, 2010.
9. Kowalski MP, Dubouix-Bourandy A, Bajmoczi M, Golan DE, Zaidi T, Coutinho-Sledge YS, Gygi MP, Gygi SP, Wiemer EA and Pier GB: Host resistance to lung infection mediated by major vault protein in epithelial cells. Science 317: 130-132, 2007.

10. Ryu SJ, An HJ, Oh YS, Choi HR, Ha MK and Park SC: On the role of major vault protein in the resistance of senescent human diploid fibroblasts to apoptosis. Cell Death Differ 15: 1673-1680, 2008.

11. Lange C, Walther W, Schwabe $\mathrm{H}$ and Stein U: Cloning and initial analysis of the human multidrug resistance-related MVP/LRP gene promoter. Biochem Biophys Res Commun 278: 125-133, 2000.

12. Stein U, Bergmann S, Scheffer GL, Scheper RJ, Royer HD, Schlag PM and Walther W: YB-1 facilitates basal and 5-fluorouracil-inducible expression of the human major vault protein (MVP) gene. Oncogene 24: 3606-3618, 2005.

13. Steiner E, Holzmann K, Pirker C, Elbling L, Micksche M and Berger W: SP-transcription factors are involved in basal MVP promoter activity and its stimulation by HDAC inhibitors. Biochem Biophys Res Commun 317: 235-243, 2004.

14. Ikeda R, Iwashita K, Sumizawa T, Beppu S, Tabata S, Tajitsu Y, Shimamoto Y, Yoshida K, Furukawa T, Che XF, Yamaguchi T, Ushiyama M, Miyawaki A, Takeda Y, Yamamoto M, Zhao HY, Shibayama Y, Yamada K and Akiyama S: Hyperosmotic stress up-regulates the expression of major vault protein in SW620 human colon cancer cells. Exp Cell Res 314: 3017-3026, 2008.

15. Chuang JY, Wang YT, Yeh SH, Liu YW, Chang WC and Hung JJ: Phosphorylation by c-Jun NH2-terminal kinase 1 regulates the stability of transcription factor Sp1 during mitosis. Mol Biol Cell 19: 1139-1151, 2008.

16. Mak SK and Kültz D: Gadd45 proteins induce G2/M arrest and modulate apoptosis in kidney cells exposed to hyperosmotic stress. J Biol Chem 279: 39075-39084, 2004.

17. Kadonaga JT, Carner KR, Masiarz FR and Tjian R: Isolation of cDNA encoding transcription factor Sp1 and functional analysis of the DNA binding domain. Cell 51: 1079-1090, 1987.

18. Ramos A, Ho WC, Forte S, Dickson K, Boutilier J, Favell K and Barker PA: Hypo-osmolar stress induces p75NTR expression by activating Sp1-dependent transcription. J Neurosci 27: 1498-1506, 2007.

19. Bell LM, Leong ML, Kim B, Wang E, Park J, Hemmings BA and Firestone GL: Hyperosmotic stress stimulates promoter activity and regulates cellular utilization of the serum- and glucocorticoid-inducible protein kinase (Sgk) by a p38 MAPK-dependent pathway. J Biol Chem 275: 25262-25272, 2000.

20. Olofsson BA, Kelly CM, Kim J, Hornsby SM and AzizkhanClifford J: Phosphorylation of Sp1 in response to DNA damage by ataxia telangiectasia-mutated kinase. Mol Cancer Res 5: 1319-1330, 2007.

21. Huangfu WC, Omori E, Akira S, Matsumoto $\mathrm{K}$ and Ninomiya-Tsuji J: Osmotic stress activates the TAK1-JNK pathway while blocking TAK1-mediated NF-kappaB activation TAO2 regulates TAK1 pathways. J Biol Chem 281: 28802-28810, 2006.

22. Ho CY and Li HY: DNA damage during mitosis invokes a JNK-mediated stress response that leads to cell death. J Cell Biochem 110: 725-731, 2010.

23. Wang YT, Yang WB, Chang WC and Hung JJ: Interplay of posttranslational modifications in Sp1 mediates Sp1 stability during cell cycle progression. J Mol Biol 414: 1-14, 2011.

24. D'Addario M, Arora PD, Ellen RP and McCulloch CA: Interaction of p38 and Sp1 in a mechanical force-induced, beta 1 integrinmediated transcriptional circuit that regulates the actin-binding protein filamin-A. J Biol Chem 277: 47541-47550, 2002.

25. Lin HH, Lai SC and Chau LY: Heme oxygenase-1/carbon monoxide induces vascular endothelial growth factor expression via p38 kinase-dependent activation of Sp1. J Biol Chem 286: 3829-3838, 2011 